\title{
El discurso, el testimonio y la memoria de las víctimas desde la voz de la radio: el caso de víctimas-invíctimas*
}

\author{
DAVID SANTIAGO RINCÓN REY** \\ JENNY MARCELA RODRÍGUEZ ROJAS ${ }^{* * *}$ \\ JHON FREDY MALDONADO RUÍZ ${ }^{* \star *}$
}

* Este artículo es un aporte a la discusión en torno a el papel de los medios de comunicación masivos y los testimonios de las víctimas, a partir del proyecto de investigación "Víctimas-Invíctimas: el testimonio y la memoria desde la voz de la radio" que está avanzando el Semillero Mareiwa-2017.

** Estudiante de Comunicación Social para la Paz de la Universidad Santo Tomás, e integrante del semillero de investigación Mareiwa. Correo electrónico: davidrinconr@usantotomas.edu.co.

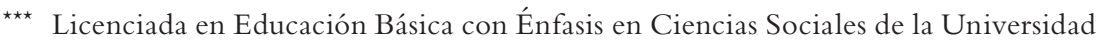
Distrital Francisco José de Caldas e Historiadora de la Universidad Nacional de Colombia, maestrante en Historia de la misma universidad. Docente del Departamento de Humanidades y Formación integral de la Universidad Santo Tomás, integrante del grupo de investigación Aletheia y líder del semillero de investigación Mareiwa. Correo electrónico: jennyrodriguezr@usantotomas.edu.co.

${ }^{\star \star \star \star}$ Licenciado en Filosofía, Ética y Valores Humanos, Especialista en Educación con Énfasis en Evaluación Educativa y Magister en Educación de la Universidad Santo Tomás. Docente del Departamento de Humanidades y Formación integral de la Universidad Santo Tomás, integrante del grupo de investigación Aletheia y co-investigador del semillero de investigación Mareiwa. Correo electrónico: jhonmaldonado@usantotomas.edu.co. 


\section{INTRODUCCIÓN}

El siguiente trabajo de investigación consiste en el análisis de los discursos por parte de fuentes no oficiales en el marco del conflicto armado, haciendo evidentes las tensiones que se presentan allí. Este análisis, ayudará a ver el conflicto histórico desde la otredad y mostrará las contradicciones con los discursos de los medios de comunicación masivos y entidades estatales.

La investigación se realizó a partir del estudio de los discursos de víctimas del conflicto armado de diferentes lugares de Colombia, quienes, en colaboración con estudiantes de cuarto semestre de la Universidad Santo Tomás, crearon el programa radial "Víctimas-Invíctimas". Este texto mediático es un espacio de reflexión, confrontación y crítica. Partimos de la necesidad de hacer un estudio sobre medios de comunicación, en donde se muestre que los discursos dominantes van en contradicción a la realidad que las víctimas cuentan, generando así la revictimización y la invisibilización de las mismas. Esto se logra con el análisis del discurso oficial, esto es, las políticas públicas y leyes enfocadas en las víctimas (en la que se destaca la ley 1448 de 2011).

El objetivo de este trabajo es evidenciar si los discursos generados por parte de entidades estatales y medios de comunicación masivos están orientados a lograr un cambio social donde se destaque a las víctimas, se reivindique la vida y la memoria de las personas que sufrieron la violencia en Colombia, o si por el contrario, se hace necesario crear espacios como el de Victimas In-Víctimas con el propósito hacer resistencia frente a la revictimización que ofrecen los medios masivos de comunicación y los discursos estatales.

\section{MARCO TEÓRICO}

Pensar y actuar sobre los diferentes escenarios y personas que han estado inmersas en el conflicto, es uno de los retos a los que debemos dirigir la mirada presente y futura. Lejos está actuar teniendo como eje central "protagonistas", esto es, personas de uno u otro "bando" que han tenido la oportunidad de presentar -o ser presentados- en medios de comunicación su papel o percepción del conflicto nacional.Ya el cine colombiano había dado unos primeros pasos para pensar ese subalterno, particularmente Lisandro Duque (2008) con la película Los actores del conflicto había 
develado dos factores que consideramos resaltar: 1) la fuerza del discurso y el impacto que se puede tener a través de escenarios como los noticieros televisivos y 2) que hay historias subrepticias que es necesario develar.

En ese sentido compartimos la conceptualización de David Buckingham (2015), quien comprende los medios1 como un escenario que, a través de sus discursos, no muestra el mundo de forma transparente, sino que solo hace visible las cosas que le convienen. Así pues, al hablar de las víctimas del conflicto armado, muchos de estos medios masivos, privilegian la voz de solo un sector, aquel que se quiere exaltar. Esto, claro, dependiendo del interés. Así se observa, por ejemplo, los minutos dedicados a políticos e incluso militares frente a campesinos e indígenas, logrando así, manipular a la audiencia con la prevalencia de un solo discurso.

Por ello, resulta pertinente comprender y rescatar la alteridad para abordar la diferencia con la que cada ser, como individuo, se relaciona. Particularmente, desde tres de los cinco planos2 que Olaya Fernández (2015) expone sobre la obra del filósofo Levinas, siendo estos el plano individual, el plano intersubjetivo y el plano ético.

Plano individual: En otro nivel, se asume que la alteridad forma parte de la propia identidad, en tanto que aquello que nos particulariza y describe como individuos, lo que denominamos personalidad o carácter, es precisamente una suma de acontecimientos heterogéneos, una síntesis de todos los momentos vividos y experimentados de forma concreta, única e intransferible.

Plano intersubjetivo: a través del lenguaje nos abrimos a la alteridad, la palabra siempre es una interpelación que el otro me lanza y a la que yo respondo o, al menos, tengo el imperativo ético de responder -ambas dimensiones, la ética y la lingüística, están estrechamente conectadas en los planteamientos de Levinas-. Esa respuesta supone, ya desde el

1 Comprendiendo medios como "todo el abanico de los medios modernos de comunicación social: televisión, cine, vídeo, radio, fotografía, publicidad, periódicos y revistas, música grabada e internet" Buckingham (2015, p.19).

2 Los planos propuestos son el metafísico, religioso, individual, intersubjetivo y el plano ético (Fernández, 2015, p. 424). 
comienzo, un intento de comprender su alteridad, de acercarme a ella sin anularla. Plano ético: capto al otro como alteridad que no poseo ni puedo poseer, y esto me induce a respetar al otro en su diferencia y especificidad. La ética aflora, pues, de la confrontación directa con el rostro del otro y la actitud receptiva frente a la interpelación directa que ese rostro me lanza. En el plano ético confluyen todos los demás, ya que a través de la toma de conciencia de la alteridad del otro, y de mi propia alteridad constitutiva, comienza un nuevo proyecto de relación interpersonal basado en el diálogo, el respeto, la tolerancia, y la aceptación de la diferencia -y no solo de la semejanza-. (Olaya Fernández, 2015, p. 424)

La alteridad es entonces fundamental para esta investigación, porque permite eliminar las falsas percepciones que se tiene del otro y entablar un diálogo donde se le dé participación, "el otro no es un objeto ante el que deba adoptarse la posición de sujeto, no es algo que se pueda poseer o dominar o controlar, sino que es, ante todo y sobre todo, otro que merece mi respeto, y cuya libertad debe defender en igual medida que defiendo mi propia libertad" (Fernández, 2015, p. 424).Ya que el propósito del programa Victimas-Invíctimas es generar discursos desde la otredad y la alteridad, resulta pertinente para nosotros ver los alcances y las posibilidades de la propuesta.

Comprendiendo que Víctimas-Invíctimas es un texto mediático, los discursos que allí se emiten también tienen una intencionalidad, por ello nos interesa hacer un análisis de ellos tomando una orientación foucaultiana. Es decir, comprendiendo el discurso como una fuerza que posee la palabra, que nace de la inquietud y permanece en el tiempo y por lo tanto es de gran importancia. Si bien, los discursos los puede producir cualquier persona, no todos son conocidos, pues dependen del medio por el cual se difundan. Víctimas-Invíctimas propicia un discurso valorativo, esto es, "analiza los discursos de valor, los paradigmas sociales de evaluación a partir de los cuales los lectores generan sus juicios, las estructuras institucionales a través de las cuales el valor se forma, se transmite y se regula" (Maristany, sf, p. 188). Además, siguiendo la línea del discurso, los planteamientos de Van Dijk sobre la crítica de los abusos de poder social, el dominio y la desigualdad, generados desde el discurso, asumiendo posiciones explícitas en los asuntos y combates políticos resultan importantes, puesto que el discurso generado 
por los participantes del programa radial son discursos con una problemática social, además, son históricos, son interpretativos y explicativos, los anteriores, son principios básicos para hacer un análisis crítico del discurso.

Este discurso está fundamentado, al menos para el texto mediático, en el testimonio, entendiéndolo como una de las formas de resistencia que ha aparecido a lo largo de la historia, pues su significado es "aquel que da fe de algo" lo cual implica que esta persona tiene que haber vivido algo para dar un testimonio, también implica que ese testimonio debe ser una verdad y que es parte de la memoria y, según el autor, incluso un arte de la misma. El testimonio entonces se convertiría en la primera herramienta con la cual se crea la memoria y luego la narración. La memoria se narra.

Finalmente, dos consideraciones importantes: comprendemos víctima bajo el concepto dado por Tzvetan Todorov (2013). Esto es, como aquella que al sufrir tiene derecho a hacer su propia construcción de la historia a partir de sus narrativas. De esta forma, Todorov resalta la importancia que tienen las víctimas en la construcción de memoria por medio de narrativas que permitan crear un escenario de recopilación de los hechos

\section{ANÁLISIS DE LA SITUACIÓN}

El día 26 de septiembre del año 2016, el gobierno colombiano, luego de un extenuante proceso, firmó un acuerdo de paz con las Fuerzas Armadas Revolucionarias de Colombia (FARC) para dar fin a un conflicto de más de tres décadas; esto con el objetivo a mediano y largo plazo de propiciar una cultura de paz y reconciliación en la que hubiese una reparación a las víctimas y un compromiso de no repetición de los hechos atroces que han marcado la historia de nuestro país.

A pesar de que existe un acuerdo de paz con las FARC, firmado y revisado por entidades internacionales, aún existen otros grupos que siguen propiciando la violencia. Además de esto, el país continúa en una situación de injusticia social, acrecentado por los recientes casos de corrupción mostrados ante la opinión pública. Lo anterior, exalta otros tipos de violencia que no tienen nada que ver con el conflicto armado.

A raíz de todos los delitos y violaciones de los derechos humanos cometidos en medio de la guerra (desplazamiento forzado, atentados, violaciones, 
crímenes de Estado, despojo de tierras, secuestros, extorsiones), se crearon grupos de víctimas del conflicto, que tienen como objetivo luchar por la justicia, la reparación, la verdad y la no repetición de estos hechos que dejaron a muchos sin hogar, sin familia y los obligaron a emigrar hacia otras ciudades, como la capital del país, para intentar empezar de nuevo.

Uno de los escenarios que han adquirido mayor protagonismo es el relacionado con los medios de comunicación, particularmente en lo referido a la visibilización de las diferentes víctimas del conflicto colombiano. Por ejemplo, las palabras de Alejandra Gaviria, hija del dirigente de la Unión Patriótica Francisco Gaviria, el 9 de abril de 2017, día de conmemoración a las víctimas en el Congreso de la República, después de que el senador Álvaro UribeVélez se retirara de dicho recinto, por no recibir el beneficio de la réplica y que todos los periodistas salieron tras el senador evitado así, las intervenciones de las víctimas:

\footnotetext{
No puede ser que un congresista venga y desbarate la audiencia que era en memoria de las víctimas, que era para dignificar a las víctimas.Y ustedes, todos los periodistas llegan y lo entrevistan a él (Senador Álvaro Uribe Vélez) cuando no es capaz de respetar el uso de la palabra (...) Yo les recomiendo a ustedes (los periodistas) que busquen otras fuentes, que esperen a la gente de las regiones (...). (Colprensa, 2017)
}

La periodista Gloria Castrillón de Colombia 20/20 de El Espectador, menciona que los problemas de los grandes medios son, en primer lugar, la falta de compromiso con la verdad, pues sólo se fian de fuentes oficiales, y dejan de lado la voz de las personas que realmente están viviendo el conflicto, además, existe cierto temor por la violencia en contra de los periodistas, lo cual no permite que estos investiguen las verdades de las zonas vulnerables de Colombia, y tercero, que los periodistas no se capacitan para hablar y trabajar con víctimas, recurren mucho en la revictimización, causando así una imagen negativa de estos. Esto solo logra que la sociedad conciba a la población víctima como inferior, como la otredad.

Uno de los ejemplos más claros de tergiversación de la información por parte de los medios es el caso de Sigifredo López, quien acusó al programa La Noche del canal $R C N$, de difundir un video donde se le acusaba 
de haber negociado con las FARC planeando la ejecución de los demás diputados del valle, argumento que fue desmentido por el cabecilla de la guerrilla "Pablo Catatumbo".

Para Ligia Monroy, quien es víctima del conflicto armado en el departamento del Huila, le es muy complicado demostrar los vejámenes cometidos por ambos bandos durante el conflicto. Las pruebas que tiene del desplazamiento y los atentados que sufrió no son válidos para la Unidad de Víctimas y mucho menos para la Fiscalía, los beneficios que debería tener por estos hechos son mínimos. En la sección Hoy Soy Paz del programa Víctimas Invíctimas, Monroy denuncia la falta de organización de la Unidad deVíctimas y lo que es aún más grave, la vinculación de personas externas al conflicto, quienes se hacen pasar por víctimas con la intención de obtener beneficios, aprovechando la desorganización de la entidad y la crisis judicial por la que está pasando el director de la Unidad deVíctimas Alan Jara.

Una última voz, la da Lorena Gaitán, antropóloga y perteneciente al MOVICE, ella advierte que aún falta un compromiso pleno por parte de las instituciones gubernamentales y medios de comunicación, por lo cual, proyectos como Victimas Invíctimas son la oportunidad de escuchar las voces de las minorías, las voces que no han sido escuchadas pero que crean memoria, que crean historia.

Todo lo anterior, busca crear cambios en la sociedad, en primera parte, el programa radial es un espacio donde se da la oportunidad a las víctimas de denunciar las inconformidades, con el fin de que se mejore la calidad de vida de las mismas y se hagan valer sus derechos. En segunda parte, es una resistencia contra los discursos generados por los medios de comunicación y además, una invitación para que tengan en cuenta a las fuentes no oficiales, sin tener la necesidad de tergiversar la verdad. Para finalizar, en tercera parte, es la forma en la que se reconstruye la memoria y la historia, la cual es una garantía de no repetición, pues saber de la realidad del país involucra a todos y hay que mirarlas desde diferentes perspectivas.

\section{ANÁLISIS DE RESULTADOS}

La problemática de la revictimización de las víctimas del conflicto interno armado en Colombia por parte de los discursos generados por los medios 
masivos de comunicación, está enfocada en que en estos espacios priman los testimonios de los entes gubernamentales y las instituciones que pertenecen al Estado, sin tener en cuenta a los que verdaderamente están implicados en el conflicto, esto es, quienes padecieron el conflicto directamente.

Para víctimas como Laura Abraem Martínez, sobreviviente del conflicto armado del Guainía afirma que los medios de comunicación situaron a quienes han sido afectados por la guerra como una versión contemporánea de la novela de Víctor Hugo "Los Miserables" (1862), teniendo en cuenta que las grandes compañías de prensa en Colombia, que manejan la información a su acomodo, difundiendo mensajes que solo revictimizan a las víctimas, sin mencionar que en las ciudades, dichos mensajes se reproducen cotidianamente entre la gente que ha vivido el conflicto indirectamente. Para Martínez, el papel de los medios es dignificar a las personas sobrevivientes del conflicto, sin crear imaginarios sobre ellos a la sociedad. Además, afirma que los medios deben hablar por la ciudadanía como forma de hacer valer los derechos humanos de las personas y exigir que entidades gubernamentales hagan lo mismo. Sin embargo, eso no sucede.

Una de las problemáticas que denuncian los integrantes de Víctimas-Invíctimas es en contra de las entidades estatales, que deben reparar a las víctimas y hacer cumplir sus derechos, por ejemplo, la Unidad deVíctimas, la cual tiene que velar por los derechos de las personas que estaban en medio del conflicto, regidos por la ley 1448 de 2011, la cual brindó al Presidente de la República facultades extraordinarias para expedir, por medio de decretos con fuerza de ley, la regulación de los derechos y garantías de las víctimas pertenecientes a los pueblos indígenas, comunidades Rom y comunidades negras (afrocolombianas, raizales y palenqueras).

Otro testimonio de abuso de poder es dado por Oscar Gómez, locutor del programa radial Víctimas-Invíctimas, y sobreviviente del conflicto armado en Barrancabermeja. Para él, el Estado está incumpliendo a las víctimas, esto mirado a través de la ley 1448 de 2011, que en su artículo 3 dice

se consideran víctimas, para los efectos de esta ley, aquellas personas que individual o colectivamente hayan sufrido un daño por hechos ocurridos a partir del $1^{\circ}$ de enero de 1985 , como consecuencia de infracciones 
al Derecho Internacional Humanitario o de violaciones graves y manifiestas a las normas internacionales de Derechos Humanos, ocurridas con ocasión del conflicto armado interno. (Ley 1448 de 2011)

Sin embargo, esta ley no menciona de los problemas que tienen las víctimas para evidenciar que efectivamente fueron afectados por el conflicto. "Existen personas que lo perdieron todo, que no pueden volver a los lugares de los que salieron porque la violencia sigue presente allí. ¿Cómo uno puede dar veracidad de que sí es una víctima?” mencionó Oscar Gómez en 2016.

\section{CONCLUSIONES}

Lejos está aún fijar una o algunas conclusiones frente al tema de la victimización a través de los medios de comunicación. La cercanía con el hecho estructural, esto es, la firma de los acuerdos de paz, y el desescalamiento del conflicto están en una fase inicial, el día a día puede marcar diferencias. Lo que sí es claro es la posibilidad de generar espacios de paz, este es el principal aporte de Victimas-Invíctimas, jóvenes que desde su profesionalización construyen y convocan diversos protagonistas, pero más importante, diversos testimonios, más allá de la búsqueda de una audiencia, más allá de los discursos hegemónicos y preponderantes, la voz que literalmente de las víctimas a otras víctimas del conflicto armado en Colombia permitirán conocer discursos, nuevas formas de comprender el mundo, pero mejor aún, nuevas herramientas para actuar en este mundo.

Con todo lo anterior, mantener una memoria verídica resulta una utopía y más con un país lleno de situaciones violentas como la colombiana.Y más cuando son los medios, quienes son los encargados de narrar los hechos a lo largo y ancho del país. Los medios masivos de comunicación le dan más prioridad a las fuentes oficiales, quienes, con un discurso político, crean memoria con base a sus intereses, sin embargo, jamás se le da oportunidad de hablar a lo que Homi Bhabha (1994) llama como otredad, es decir, aquello que es invisibilizado por las grandes hegemonías, logrando así crear una falsa memoria colectiva.

Por lo anterior, surge la pregunta ¿por qué la marginación de las fuentes no oficiales? Para Enrique Santos (2004), uno de los principales factores 
por los cuales los medios no llegan a las zonas donde habitan los sobrevivientes del conflicto, es la intimidación por parte de los grupos subversivos contra la prensa. Santos afirma que los crímenes cometidos contra periodistas son casos extremadamente impunes, lo cual ha generado que los medios le den prioridad a las fuentes oficiales, perdiendo así la objetividad y la transparencia. Esto ha ocasionado que mientras que en las zonas rurales los medios de comunicación alternativos como Nueva Colombia Noticias, donde los discursos claramente son políticos, en la urbanidad sean los medios los causantes de que las memorias que el conflicto ha dejado, desde la perspectiva de las personas que vivieron el conflicto de manera directa, se mantengan olvidadas.

Ahora bien, uno de los problemas de todo lo anterior, Todorov lo define como "abusos de memoria", donde el autor se opone a la manipulación de la memoria colectiva con el propósito de catalogarlo como una categoría general, la cual no da espacio para una concientización y volviendo la experiencia transitiva, generando que las personas se vuelvan indolentes e indiferentes con la historia, la misma que debe aprenderse para saber las causas de los hechos violentos, de los efectos de dichos sucesos y de cómo afrontar las crisis políticas, económicas, sociales y culturales.

Por lo anterior, es importante seguir pendiente del programa radial Víctimas-Invíctimas cuya voz principal es la de víctimas de desplazamiento, atentados y otros vejámenes cometidos por todos los bandos del conflicto armado. Este grupo de personas usan el programa de radio como resistencia, crítica y denuncia contra la negligencia del Estado y contra la invisibilización social provocada por los medios de comunicación masiva.

\section{REFERENCIAS}

Bhabha, H. (2004). El lugar de la cultura. Buenos Aires, Argentina: Routledge

Barranquero-Carretero, A. y Sáez Baeza, C. (2010). Comunicación Alternativa y comunicación para el cambio social democrático. Revista: Comunicación y desarrollo en la era digital. Congreso AE-IC 3, 4 y 5 de febrero de 2010, (271) 1-21 
Buckingham, D. (2005). Educación en medios: alfabetización, aprendizaje y cultura contemporánea. Barcelona, España: Ediciones Paidós ibérica S.A.

Cardona Álzate, J., Castrillón Pulido, G., Morelo Martínez, G., García, K. A. y Bahar Leiser, O. (2016). Pistas Para Narrar La Memoria. Bogotá, Colombia: Konrad-Adenauer-Stiftung e.V., KAS.

Caro, S. J. (2014). Equipo Paz Gobierno. Recuperado de Equipo Paz Gobierno: http:// equipopazgobierno.presidencia.gov.co/prensa/declaraciones/Paginas/paz-territorial-sergio-jaramillo- alto-comisionado- paz-proceso- paz.aspx

Centro de Memoria histórica, (2013) iBasta ya; Memorias de guerra y dignidad. Versión virtual. Recuperado de: www.centrodememoriahistorica.gov.co/micrositios/ infor meGeneral/descargas.html

Jarque, C. M. y Medina, F. (1998). Índices de Desarrollo Humano en México 1960-1990. Santiago de Chile: Cepal (Comisión Económica para América Latina).

Fernández, O. (2014). "La antropología de Unamuno: el 'hombre de carne y hueso"”, Aragüés y Ezquerra (coords.). De Heidegger al postestructuralismo.Panorama de la ontología y antropología contemporáneas. Zaragoza, Prensas de la Universidad de Zaragoza, pp. 71-87.

Frow, J. (1995). Cultural Studies; CulturalValue. Recuperado http://www.biblioteca. unlpam.edu.ar/pubpdf/anclajes/v01a16maristany.pdf

Foucault, M. (2016) “El orden del discurso”. Revista Hemisféricos y Polares Recuperado de: http://www.revistaestudioshemisfericosypolares.cl/ articulos/108-Manzano-Revista_Estudios_Hemisfericos_y_Polares_Vol_7_N_3_ (Julio-Septiembre_2017).pdf

Lévinas, Emmanuel. (2004). El tiempo y el otro, Barcelona, España: Paidós.

Congreso de la República. Ley 1448 de 2011. Por la cual se dictan medidas de atención, asistencia y reparación integral a las víctimas del conflicto armado interno y se dictan otras disposiciones. Recuperado de http://servicios.minminas.gov.co/compilacionnormativa/docs/pdf/ley_1448_2011.pdf

Martínez, C. (2013). Ley de víctimas y restitución de tierras en Colombia en contexto. Berlín, Alemania: Forschungs- und Dokumentationszentrum Chile-Lateinamerika - FDCL e.V

Muñoz-González, R. (2016). Más allá de la sangre: procesos de revictimización y periodismo sensacionalista Estudios sobre el Mensaje Periodístico, 22 (2), 829-845.

Reyes, F., Suárez, M., Herrera, M. (2016) La comunicación en un eventual escenario de transición y posconflicto. Bogotá, Colombia: Ediciones USTA. 
Thompson, J. (1995). The media and modernity. A social theory of the media. Stanford, CA: Paidós.

Todorov, T. (2013). Los usos de la memoria. Dossier, [online] (10), pp.4-10. Recuperado de: http://idehpucp.pucp.edu.pe/wp-content/uploads/2012/09/Todorov.pdf 